\title{
FIXED POINT APPROXIMATIONS FOR NORM-ATTAINABLE OPERATORS
}

\author{
BENARD OKELO \\ Department of Pure and Applied Mathematics, \\ Jaramogi Oginga Odinga University of Science and Technology, \\ Box 210-40601, Bondo-Kenya \\ Corresponding author: bnyaare@yahoo.com \\ Received Oct. 1, 2020
}

\begin{abstract}
Aвstract. Fixed points approximation is a very important aspect of complex Hilbert spaces in fixed point theory. This paper discusses approximation of nonexpansive operators on Hilbert spaces in terms of fixed points especially when the operators are norm-attainable. In particular, we prove that for an invariant subspace $H_{0}$ of a complex Hilbert space $H$, there exists a unique nonexpansive retraction $R$ of $H_{0}$ onto $\Xi(\mathcal{Q})$ and $x \in H_{0}$ such that the sequence $\left\{\xi_{n}\right\}$ generated by $\xi_{n}=\epsilon_{n} f\left(\xi_{n}\right)+\left(1-\epsilon_{n}\right) T_{\xi_{n}} \xi_{n}$ is strongly convergent to $R x$ for all $n \in \mathcal{N}$.
\end{abstract}

2010 Mathematics Subject Classification. 47B47; 47A30.

Key words and phrases. fixed point; norm-attainability; Hilbert space; nonexpansivity.

\section{INTRODUCTION}

A lot of studies involving mappings on Banach spaces have been done over along period of time with interesting results obtained(see [2] - [11] and the references therein). On fixed point theory in convex sets in particular, characterizations have been carried out with nice expositions [12]. The interesting aspect is that, provided the existence of a fixed point of a given mapping has been found, what remains is to determine the value of that fixed point which is not a trivial task [12]. This is the reason why iterative processes are put into action for computing them. The Banach contraction theorem [1] utilized Picard iteration process in approximating a fixed point. This work characterizes nonexpansive contractions in normattainable classes. Here is the main theorem of this work.

DOI: 10.28924/APJM/8-3 
Theorem 1.1. Let $\mathcal{Q}$ be a two-sided maximal ideal of a real separable Hilbert space $H$ and $H_{0}$ be a reflexive invariant subspace of $H$. Suppose that $P=\left\{T_{s}: s \in \mathcal{Q}\right\}$ is a canonical representation of $\mathcal{Q}$ from $H$ into itself such that the essential closure of $\left\{T_{t} x: t \in \mathcal{Q}\right\}$ is sequentially compact for every $x \in H_{0}$ and $\Xi(\mathcal{Q}) \neq \emptyset$. Suppose that $X$ is an invariant subspace of $N A(\mathcal{Q})$ such that $1 \in X, t \mapsto\left\langle T_{t} x, x^{*}\right\rangle$ is an element of $X$ for each $x \in H_{0}$ and $x^{*} \in H^{*}$. Consider $\left\{\gamma_{n}\right\}$ as monotone increasing sequence of $X$. Suppose that $f$ is a contraction on $H_{0}$. Let $\epsilon_{n}$ be a sequence in $(0,1)$ such that $\lim _{n} \epsilon_{n}=0$. Consider the duality mapping $J$ to be weakly sequentially continuous. Then we have a unique nonexpansive retraction $R$ of $H_{0}$ onto $\Xi(\mathcal{Q})$ and $x \in H_{0}$ such that the sequence $\left\{\xi_{n}\right\}$ generated by $\xi_{n}=\epsilon_{n} f\left(\xi_{n}\right)+\left(1-\epsilon_{n}\right) T_{\xi_{n}} \xi_{n}$ is strongly convergent to $R x$ for all $n \in \mathcal{N}$.

We note that an operator $S \in B(H)$ is said to be norm-attainable if there exists a unit vector $x \in H$ such that $\|S x\|=\|S\|$. We denote the class of all norm-attainable operators on $H$ by $N A(H)$. For $S \in N A(H)$, we call $x$ a fixed point of $S$ if $S(x)=x$. If $H_{0}$ is an invariant subspace of $H$ then $S \in N A(H)$ is said to be nonexpansive if $\|S x-S y\| \leq\|x-y\|$, for all $x, y \in H_{0}$. In the next section we give the main results in this note.

\section{MAIN Results}

Now we give the main results of this study. First, we begin with some auxiliary results and we express our conditions by removing the compactness condition on an invariant subspace $H_{0}$ of a complex Hilbert space $H$ as follows.

Proposition 2.1. Let $H_{0}$ be an invariant subspace of $H$. For every two-sided maximal ideal $\mathcal{Q}$ of complex Hilbert space $H$, there exists a unique nonexpansive retraction $\Xi(\mathcal{Q})$ of $H_{0}$.

Proof. Since $H$ has the Opial's condition then by Banach contraction mapping theorem, we can fix a sequence $\xi_{n}$ in $H_{0}$ i.e. $\xi_{n}=\frac{1}{n} x+\left(1-\frac{1}{n}\right) T_{\xi} \xi_{n} \quad(n \in \mathcal{N})$, where $x \in H_{0}$ is fixed and $\xi$ is an invariant on $X$. From [2], we have $\lim _{n \rightarrow \infty}\left\|\xi_{n}-T_{\xi} \xi_{n}\right\|=0$. The boundedness of $\left\{\xi_{n}\right\}$ is trivial so we that it weakly converges to an element of $\Xi(\mathcal{Q})$. That is, we prove that the weak limit set of $\left\{\xi_{n}\right\}$ denoted by $\omega_{\omega}\left\{\xi_{n}\right\}$ is contained in $\Xi(\mathcal{Q})$. Let $x^{*} \in \omega_{\omega}\left\{\xi_{n}\right\}$ and consider $\left\{\xi_{n_{j}}\right\}$ be a subsequence of $\left\{\xi_{n}\right\}$ such that $\xi_{n_{j}} \rightarrow x^{*}$. Since $I-T_{t}$ is semiclosed at diminishing point, for each $t \in \mathcal{Q}$, then we conclude that $x^{*} \in \Xi(\mathcal{Q})$. Therefore, $\omega_{\omega}\left\{z_{n}\right\} \subseteq \Xi(\mathcal{Q})$. Now $\left\{\xi_{n}\right\}$ is bounded and $H$ is separable, so $\left\{\xi_{n}\right\}$ is a sequentially compact subset of $H$, hence we have $\left\{\xi_{n_{j}}\right\}$ of $\left\{\xi_{n}\right\}$ such that $\left\{\xi_{n_{j}}\right\}$ sequentially converges to a point $\xi$. Invoking nonexpansivity of retractions of $H_{0}$ onto $\Xi(\mathcal{Q})$, uniqueness is proved and the proof is complete. 
The next result is an analogy of of known assertions whereby we remove the compactness condition on $H_{0}$ for reflexive real separable Hilbert spaces as follows.

Lemma 2.1. For every two-sided maximal ideal $\mathcal{Q}$ of an infinite dimensional, reflexive, separable Hilbert space $H$, let $X$ be a left invariant subspace of $N A(\mathcal{Q})$ such that $1 \in X$, and the function $t \mapsto\left\langle T_{t} x, x^{*}\right\rangle$ is an element of $X$ for each $x \in H_{0}$ and $x^{*} \in H^{*}$. Then there exists a unique nonexpansive retraction $\Xi(\mathcal{Q})$ of $H_{0}$

Proof. Since $H$ has the Opial's condition, let $x \in H_{0}$. We fix the following set $D=\left\{y \in H_{0}\right.$ : $\|y-p\| \leq\|x-p\|\}$. Since $D$ is reflexive and $x \in D$ we have $T_{t}(D) \subset D$. Given $\epsilon>0$, the rest of the proof follows immediately from Proposition 2.1. This completes the proof.

Now we dedicate our efforts to proving Theorem 1.1.

Proof. We proceed with the Proof of Theorem 1.1 as follows. We know that $H$ has the Opial's condition and that $H$ is a reflexive real separable Hilbert space. We give our proof in five steps as illustrated below.

Step $(i)$. Existence of $\left\{\xi_{n}\right\}$. This is guaranteed from Proposition 2.1 and also follows Step 1 of [2].

Step $(i i) .\left\{\xi_{n}\right\}$ is bounded. To see this, let $p \in \Xi(\mathcal{Q})$. Since $T_{\xi_{n}} p=p$ for each $n \in \mathcal{N}$, by simple calculation we have $\left\|\xi_{n}-p\right\|^{2} \leq \epsilon_{n} \alpha\left\|\xi_{n}-p\right\|^{2}+\left(1-\epsilon_{n}\right)\left\|\xi_{n}-p\right\|^{2}+\epsilon_{n}\left\langle f(p)-p, J\left(\xi_{n}-p\right)\right\rangle \leq$ $\frac{1}{1-\alpha}\left\langle f(p)-p, J\left(z_{n}-p\right)\right\rangle$. So, $\left\|z_{n}-p\right\| \leq \frac{1}{1-\alpha}\|f(p)-p\|$, proving the boundedness of $\left\{\xi_{n}\right\}$. Step(iii). We show that $\lim _{n \rightarrow \infty}\left\|\xi_{n}-T_{t} z_{n}\right\|=0$, for all $t \in \mathcal{Q}$. To see this, consider $t \in \mathcal{Q}$. Let $p$ be an arbitrary element of $\Xi(\mathcal{Q})$. Set $D=\left\{y \in H_{0}:\|y-p\| \leq \frac{1}{1-\alpha}\|f(p)-p\|\right\}$. From the statement of the theorem, we know that that $D$ is reflexive and $\left\{\xi_{n}\right\} \subset D$ and $T_{t}(D) \subset D$. The rest of the proof follows from step (vii) in [12].

$\operatorname{Step}(i v)$. We have a unique retraction $R$ of $H_{0}$ onto $\Xi(\mathcal{Q})$ and $x \in H_{0}$ such that $\Gamma:=\lim _{\sup _{n}}\langle x-$ $\left.R x, J\left(\xi_{n}-R x\right)\right\rangle \leq 0$. The proof follows from the definition of $\Gamma$ and from Step (ii) that $\left\{\xi_{n}\right\}$ is bounded, and since $H$ is reflexive and separable, so we have $\left\{\xi_{n_{j}}\right\}$ of $\left\{\xi_{n}\right\}$ satisfying $\lim _{j}\left\langle x-R x, J\left(z_{n_{j}}-R x\right)\right\rangle=\Gamma$ and $\left\{\xi_{n_{j}}\right\}$ sequentially converges to a point $\xi$. By considering Lemma 2.1, and the definition of nonexpansive operator the rest is clear.

$\operatorname{Step}(v) .\left\{\xi_{n}\right\}$ strongly converges to $R x$. Indeed, this follows from the fact that $H$ is separable and $\lim \sup _{n}\left\|\xi_{n}-R x\right\|^{2} \leq \frac{2}{1-\alpha} \lim \sup _{n}\left\langle x-R x, J\left(\xi_{n}-R x\right)\right\rangle \leq 0$. By simple manipulation it is easy to see that $\xi_{n} \rightarrow R x$. This completes the proof. 


\section{CONCLUSION AND OPEN PROBLEM}

In this work, we have proven that for an invariant subspace $H_{0}$ of a complex Hilbert space $H$, there exists a unique nonexpansive retraction $R$ of $H_{0}$ onto $\Xi(\mathcal{Q})$ and $x \in H_{0}$ such that the sequence $\left\{\xi_{n}\right\}$ generated by $\xi_{n}=\epsilon_{n} f\left(\xi_{n}\right)+\left(1-\epsilon_{n}\right) T_{\xi_{n}} \xi_{n}$ is strongly convergent to $R x$ for all $n \in \mathcal{N}$.The open question therefore is: Does Theorem 1.1 hold for Banach spaces in general?

\section{ACKNOWLEDGEMENT}

The author is grateful to the referees for their careful reading of the manuscript and valuable comments. The author thanks the help from the editor too.

\section{AUTHOR's CONTRIBUTION}

The author contributed wholly in writing this article and declares no conflict of interest.

\section{COMPETING INTERESTS}

The author(s) declare(s) that there is no conflict of interest regarding the publication of this paper.

\section{REFERENCES}

[1] P. R. Halmos, A Hilbert space problem book, Van Nostrand, New York, 1967.

[2] N. B. Okelo, On Convex Optimization in Hilbert Spaces, Maltepe J. Math. 2 (2019), 89-95.

[3] N. B. Okelo, J. O. Agure and D. O. Ambogo, Norms of elementary operators and characterization of norm-attainable operators, Int. J. Math. Anal. 24 (2010), 1197-1204.

[4] N. B. Okelo, The norm-attainability of some elementary operators, Appl. Math. E-Notes. 13 (2013), 1-7.

[5] N. B. Okelo, $\alpha$-Supraposinormality of operators in dense norm-attainable classes, Univ. J. Math. Appl. 2 (2019), 42-43.

[6] N. B. Okelo, On orthogonality of elementary operators in norm-attainable classes, Taiwan. J. Math. 24 (2020), 119-130.

[7] N. B. Okelo, M. O. Okongo and S. A. Nyakiti, On projective tensor norm and norm-attainable $\alpha$-derivations, Int. J. Contemp. Math. Sci. 5 (2010), 1969-1975.

[8] N. B. Okelo, J. O. Agure and P. O. Oleche, Various notions of orthogonality in normed spaces, Acta Math. Sci. 33 (5) (2013), 1387-1397.

[9] N. B. Okelo, On Norm-attainable operators in Banach Spaces, J. Funct. Spaces, 2020 (2020), 8926214.

[10] N. B. Okelo, On certain conditions for convex optimization in Hilbert Spaces, Khayyam J. Math. 5 (2019), 108-112.

[11] N. B. Okelo, On Norm-Attainable Functionals in Banach Spaces, Asia Pac. J. Math. 7 (2020), 27. 
[12] S. Suantai, Y. Shehu and P. Cholamjiak, Nonlinear iterative methods for solving the split common null point problem in Banach spaces, Optim. Meth. Softw. 34 (2019), 853-874. 